\title{
Underground Structure Inspection in Geological Groundwater Environment Base on Geologic Radar Technology
}

\author{
Yu Wang ${ }^{1,2}$, Zongying Shu ${ }^{1 *}$, Tianrong Huang ${ }^{1},{\text { Yongyu } \mathrm{Li}^{1} \text {, Shihua Wang }}^{1}$ \\ ${ }^{1}$ Shanghai Urban Construction Vocational College, Shanghai, 200438, China \\ ${ }^{2}$ Shanghai Institute of Technology, Shanghai, 201418, China
}

\begin{abstract}
How to accurately detect the anti-slide pile and the surrounding rock hole is the key to the correct application of the concrete quality treatment method, at the same time, it can ensure the control effect to a certain extent, and make the treatment project more economical and effective. The presence of water in the reinforced concrete layer, coupled with the void shape, and the irregular nature of the water in the hole, may be filled with air, mud, soil and other media factors, so there is considerable complexity. The number of abnormal bodies in the contrast enhancement diagram increases significantly, which is helpful for us to observe the loose area of anti-slide pile. We can find the pixel value corresponding to the feature by detecting the abnormal data of the pile one by one.
\end{abstract}

\section{Introduction}

Anti-slide pile is a kind of underground linear building, which will inevitably be used under different hydrogeological conditions during the construction process, thus forming the water-collecting corridor that most anti-slide piles often appear. Concrete irrigation and cracks are the common geological hazards in the construction of anti-slide piles and the main diseases of anti-slide piles. Therefore, it is urgent to study the geological hazards of anti-slide piles. Geological disaster inspection and prevention measures are also essential.

\section{Radar Principle and Detection Function}

\subsection{Function of Geologic Rada}

The effective detection method is to use the transmitting antenna to transmit high frequency electromagnetic wave in the direction of buried target in the form of broad band pulse. The discontinuity of the electromagnetic wave through the medium will produce reflection, and is received by the receiving antenna. By analyzing the propagation characteristics of electromagnetic waves in time and space, the spatial location, scale and physical properties of buried targets can be obtained. Electromagnetic wave propagates in medium, its path and electromagnetic wave intensity vary with the change of electrical properties and geometric shapes. The detection resolution mainly depends on pulse width and dielectric absorption characteristics. The detection depth is closely related to the center frequency [1][2].
Ground-penetrating radar technology can achieve the following goals of concrete quality of anti-slide piles:

(1) The concrete thickness of anti-slide pile and the empty space around the anti-slide pile.

(2) Detection of reinforced concrete and distribution of grilles.

(3) Cracks are distributed inside concrete and steel grilles.

How to accurately detect the anti-slide pile and the surrounding rock water hole is the key to correctly apply the concrete quality treatment method, and at the same time, it can ensure the control effect to a certain extent and make the treatment project more economical and effective. The water falling behind the reinforced concrete layer, coupled with the hollow shape and the irregular nature of the water inside the hole, may be filled with air, mud, soil and other media factors, it is complexity.

\subsection{Determine the waveform}

(1) cavity detection: dielectric constant is the air and the difference between concrete and rock, when the pile and surrounding areas have a clearing, due to differences in different media, electromagnetic geological radar reflectivity result of waveform distortion is more apparent, reflects the positive and negative wave interval, highly visible and easy to recognize, in the presence of vacant area of pile and rock, the reflection will show a complex vibration effect, the reflected wave increase, the cavity waveform is hyperbolic shape.

(2) Thickness detection: There is a big difference between the anti-slide pile and the rock in terms of composition, physical properties, dielectric constant and so on. When the wave enters the surrounding rock from the concrete, the reflected wave amplitude increases

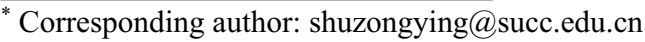


significantly and the video rate decreases. The thickness of concrete anti-slide pile structure layer is calculated according to the reflection time of electromagnetic pulse in the structure interface layer and the propagation speed of electromagnetic wave in each structure layer. The key to determine the pile thickness is to calculate the propagation time of electromagnetic wave in anti-slide pile layer.

\subsection{Enhanced wavelet technique}

In the detection of empty water of anti-slide pile, wavelet transform is used to process the image, which can not only improve the visual effect of the image and the clarity of the image elements, but also make the image become more conducive to computer processing and analysis. Common image processing techniques can be divided into image - based domain and transform domain.

How to use Wavelet-based multi-scale method for image enhancement? Firstly, we use wavelet transform technology to decomposed of image with the different size, location and orientation, and then change some of the weight coefficient with processing requirements, enhancing the interesting part, weakening other parts; Wavelet method can be decomposed into different frequency signals for variety of different frequency composition of mixed-signal, and thus can be effectively applied to the letter-noise separation, edge detection and image restoration. Here we put the brightness of the original image as $\mathrm{B}(\mathrm{x}, \mathrm{p})$, wavelet function as $\mathrm{f}(\mathrm{x}, \rho)$, then enhancing brightness

Wavelet multi-scale method is used for image enhancement. First of all, wavelet transform is used to decompose images of different sizes, positions and directions. Then, according to the processing requirements, some weight coefficients are changed to enhance the interested parts and weaken the other parts. The wavelet method can decompose mixed signals composed of different frequencies into signals of different frequencies, so it can be effectively applied to signalnoise separation, edge detection and image recovery. Here we set the brightness of the original image to $C(x, p)$, the wavelet function to $\mathrm{Q}(\mathrm{x}$,$) , and then enhance the$ brightness $\mathrm{C} /(\mathrm{x}, \rho)$ can be derived from the first two convolution".

$$
C(\bar{k})=Q(x, k) \times C(x, \bar{k})
$$

The wavelet transform is decomposed into size, position and direction in different scales, so as to selectively enlarge the amount of interest and reduce unnecessary weight. Image noise will also have high frequency information, while enhancement processing may amplify the noise. The space-frequency analysis method can enhance the edge of the image, and at the same time, the noise problem needs to be considered. Therefore, the image used should be the image that has been processed by wavelet adaptive processing. When using GPR to process images, pixel points should be added and indicated in order to detect the location of these anomalies and steel. The image "DB5" was decomposed to a secondary level, the appropriate pixel value range after transformation was selected, and the enhancement factor was selected for enhancement. The healing effect of pixels showed in Figure 1 and 2.

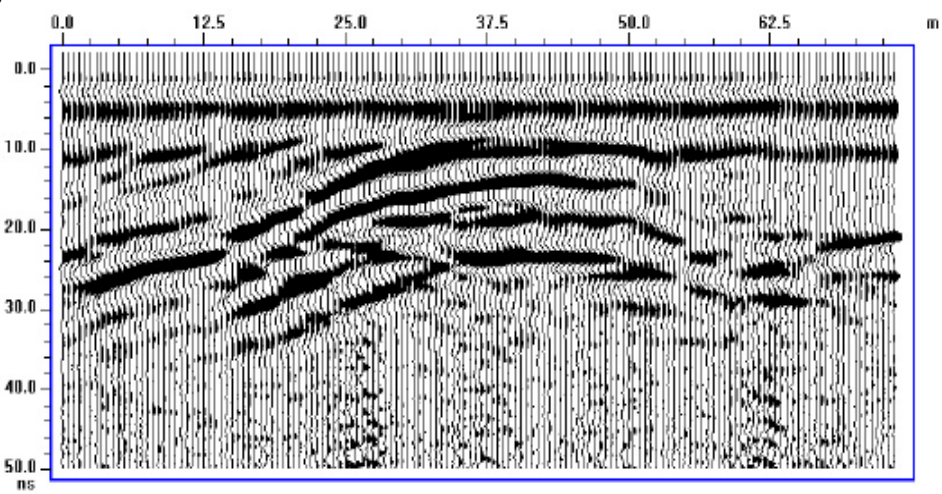

Fig 1. Comparison of Pixel Dot

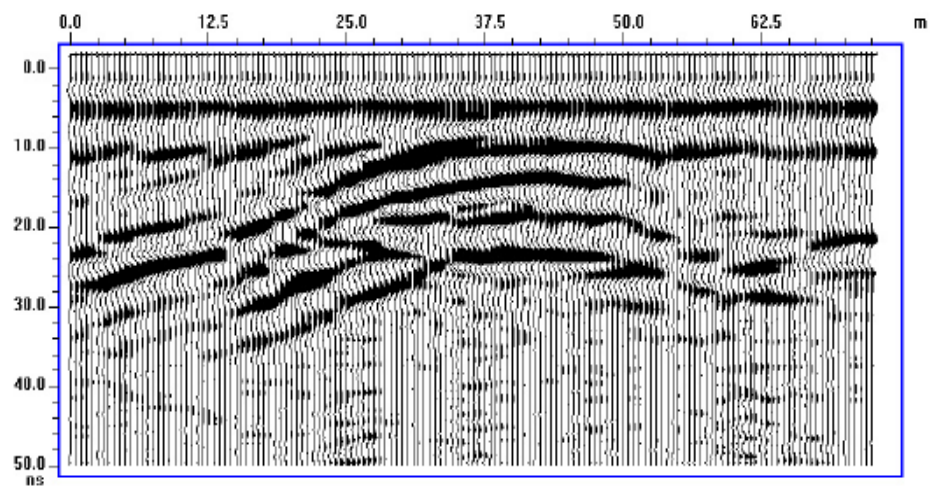

Fig 2. Comparison of Pixel Dot 
The number of abnormal bodies in the contrast enhancement diagram increases significantly, which is helpful for us to observe the loose area of anti-slide pile. We can find the pixel value corresponding to the feature by detecting the abnormal data of the pile one by one.

\section{ENGINEERING APPLICATION}

The demonstration project is for highway anti-slip structural piles around Chongqing, capital using groundpenetrating radar to survey the surrounding rocks of handdug pile foundation Bridges, to define the stability of rock documents on the foundation and external documents on the bottom foundation and the thickness of foundation rock overburden.

The sequence is composed of quaternary oblique soil belt (Qdl), quaternary residual soil belt (Qel), late Yanshan granite belt (379) and grinding wheel belt. Topographically, the site is steep and the bedrock is relatively shallow, but the terrain is very undulating. The field geological survey shows that the side walls of the pile foundation are light red and blue, accompanied by shallow, weak to micro yanshanian (446) weathered granite, partially gray-green diorite porphyry, and the rock hardness is greater than 90Mpa. When hit, it sounds clear and pleasant, full of elasticity, tentacles, not easy to cut off. The rock mass is complete, massive, and thickly layered, with two groups of joints:

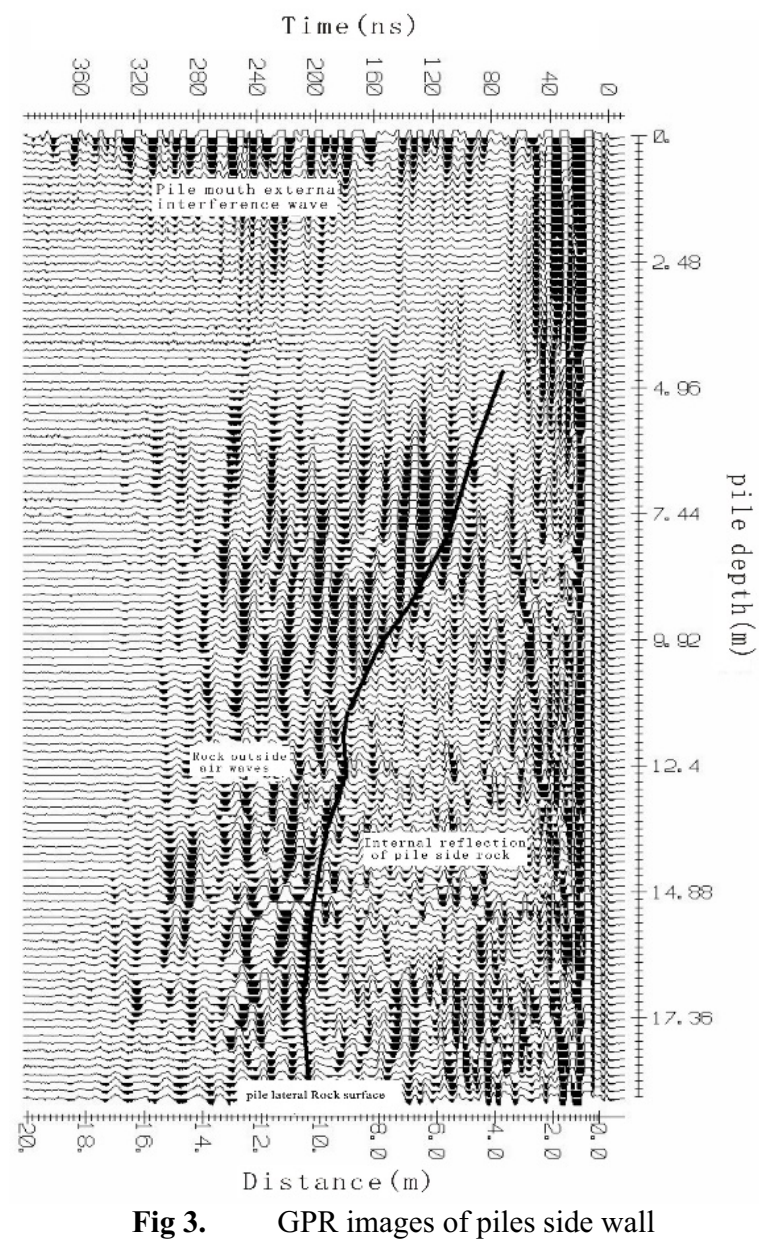

Figure 3 is the GPR record of 1-1 pile lateral wall rock mass. The cliff face boundary and internal structure are obvious, and the cliff state is steep.GPR records show that the echo energy in the surrounding rock is relatively strong (FIG. 3), and joints and fissures are developed. The spacing of the piles is from top to bottom of the rock mass outer wall from 1.9 to 9 meters, which is calculated using the travel time of the bidirectional RADA wave from the surface to reflect the rock mass and the average velocity of the radar waves passing through the rock medium. According to the lithological characteristics of the pile end, the average velocity was determined to be $0.2 \mathrm{~m} / \mathrm{ns}$, which was less than $6 \%$ different from the actual velocity of the rock medium.

\section{Conclusion}

If the surface of anti-slide pile is not smooth, especially the ground is not smooth, it will bring great difficulty to radar detection. At this point, we should observe the working condition of the antenna (whether it leaves the surface of the anti-skid pile, whether it moves to another place suddenly, etc.), and pay attention to whether the antenna post is abnormal, so as to make a correct judgment.

In addition, the radar may be quite sensitive to random interference, for example, due to steel piles and external metals in the track network. A general understanding of the interpretation of the available information, coupled 
with appropriate pit drilling or work, is required to enter the phase of quantitative interpretation.

\section{References}

1. Mallat. Characterization of signals from multiscale edges. IFEE Tans, PAMI, 1992, 4(7):711-733

2. MAO Hai-he, Application of ground probing radar to short-term geological forecast for anti-sliding piles construction[J] Rock and Soil Mechanics, 2003, s(1): 154-157

3. Xia Jiang-hai. Application of deterministic deconvolution of ground - penetrating radar data in a study of carbonate strata[J]. Journal of Applied Geophysics, 2004, 56: 213-229. 